Submitted to ICNS-97

BNL -64607

CONF-970814--

\title{
Nature of the triple point in chromium alloys: mode-softening of the incommensurate spin density wave
}

\author{
D.R. Noakes ${ }^{a}$, E. Fawcett ${ }^{b}$, B.J. Sternlieb ${ }^{c}$, G. Shirane ${ }^{c}$ \\ and J. Jankowska ${ }^{d}$ \\ ${ }^{a}$ Physics Dept., Virginia State University, Petersburg, VA 23806 \\ ${ }^{b}$ Physics Dept., University of Toronto, Toronto, ON M5S 1A7, Canada \\ ${ }^{c}$ Physics Dept., Brookhaven National Laboratory, Upton, NY 11973 \\ ${ }^{d}$ Institute for Nuclear Research SWIERK, 05-400 Otwock, Poland
}

June 29, 1997

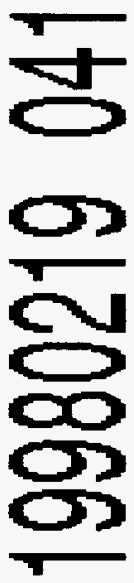

\begin{abstract}
The magnetic stiffness parameter, $\mathrm{A}^{2}$, associated with the critical fluctuations in the paramagnetic phase close to the incommensurate spin-density-wave (SDW) transition of a $\mathrm{Cr}+0.18$ at.\%Re single crystal, when compared with that of in pure chromium and dilute alloys of vanadium in chromium, indicates that the singularity responsible for the onset of the commensurate SDW phase occurs when $A^{2}$ approaches zero at a finite value of the incommensurability parameter, $\delta \cong 0.017 \mathrm{rlu}$, instead of zero as usually assumed. The mode of the incommensurate SDW softens as $\delta$ approaches this critical value with rhenium doping, and conversely hardens as $\delta$ increases when vanadium is added to chromium. Also, the amplitude of the critical scattering increases as $\delta$ approaches its end point, by a factor of about two going from 0.2 and 0.5 at.\% $\mathrm{V}$ to pure chromium, and by another factor near two in going from pure $\mathrm{Cr}$ to $\mathrm{Cr}+0.18$ at.\%Re.
\end{abstract}

Keywords: Chromium alloys, critical behavior, magnetic phase diagram, triple-axis neutron scattering

Corresponding author: David R. Noakes, Box 9325, Virginia State University, Petersburg, VA 23806. Tel. 804-524-506, FAX 804-5245439, Email: DNoakes@VSU.edu

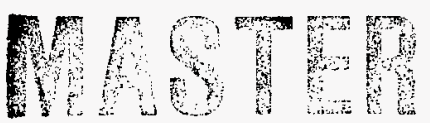


Chromium enters an incommensurate spin-density-wave (SDW) antiferromagnetic state at its Neel transition. The addition of small amounts of nonmagnetic impurity elements from the right of chromium in the periodic table generally increases the ordering temperature and makes the ordered state more nearly commensurate.[1] This is opposite to the effect of adding vanadium, which lies to the left of chromium in the periodic table, and which depresses $\mathrm{T}_{N}$ and increases the incommensurability parameter, $\delta .[2,3,4]$ Previous publications have shown that triple-axis neutron scattering observed just above the Néel temperature in single crystals of chromium and $\mathrm{Cr}(\mathrm{V})$ alloys can be analyzed in detail in terms of a magnetic critical scattering model.[5] The model assumes critical behavior associated with a second-order transition, even though there is a weak first-order transition at $\mathrm{T}_{N}$ in pure chromium. It involves an incommensurate critical susceptibility $\chi(Q, \omega)$ which intrinsically embodies the sixfold symmetry around the commensurate (010) position of the magnetic Bragg peaks that form at $\mathrm{T}_{N}$. To see if this analysis is also applicable on the other side of chromium in the magnetic phase diagram, a single crystal of $\mathrm{Cr}+0.18$ at.\%Re previously measured by Mikke and Jankowska [6] was studied at the H8 spectrometer at Brookhaven's HFBR.

The Néel transition at $\mathrm{T}_{N}=325.5 \pm 0.4 \mathrm{~K}$ to the incommensurate SDW state is first order in this sample, [7] as it is in pure chromium. To study the dynamic susceptibility, a variety of constant-energy and constant-Q scans were taken through the satellite and $(010)$ positions $10 \mathrm{~K}$ above $\mathrm{T}_{N}$. The data were analyzed by fitting the resolution-folded Sato-Maki [2] model previously used to analyze critical magnetic scattering in chromium and $\operatorname{Cr}(\mathrm{V})$ alloys. [5] The deduced values for the 


\section{DISCLAIMER}

This report was prepared as an account of work sponsored by an agency of the United States Government. Neither the United States Government nor any agency thereof, nor any of their employees, make any warranty, express or implied, or assumes any legal liability or responsibility for the accuracy, completeness, or usefulness of any information, apparatus, product, or process disclosed, or represents that its use would not infringe privately owned rights. Reference herein to any specific commercial product, process, or service by trade name, trademark, manufacturer, or otherwise does not necessarily constitute or imply its endorsement, recommendation, or favoring by the United States Government or any agency thereof. The views and opinions of authors expressed herein do not necessarily state or reflect those of the United States Government or any agency thereof. 
amplitude scale, $\chi^{0} / r^{2}$ (plotted as its reciprocal, $r^{2} / \chi^{0}$ ) and magnetic stiffness, $\mathrm{A}^{2}$, are shown in comparison to those quantities for chromium and $\mathrm{Cr}(\mathrm{V})$ alloys in the Figure, as a function of the (fit) incommensurability parameter, $\delta$. An interesting correlation is thus revealed, as indicated by the dashed lines: the magnetic stiffness appears to be tending to zero, and the amplitude scale appears to be diverging, as $\delta$ tends not to zero (the commensurate SDW), but to a finite value $\approx 0.017 \mathrm{rlu}$. This suggests that $\delta$ does not smoothly go to zero with increasing rhenium concentration, but rather that there is a range of long-wavelength critical fluctuations (and therefore SDW in the ordered phase) that is inaccessible. This would mean that there is a discontinuity in $\delta$ at the triple point in the magnetic phase diagram of the alloy system, where the Néel transition lines to the incommensurate ISDW and commensurate CSDW phases meet the ISDW-to-CSDW transition line. Such behavior is indeed found in a $\mathrm{Cr}+2.8 \% \mathrm{Fe}$ alloy, when it is taken through the triple point by applying pressure.[8]

The Figure shows that the amplitude scale $\chi^{0} / r^{2}$ seems to vary as the inverse of $\mathrm{A}^{2}$ when the alloy composition varies, i.e., the amplitude diverges while the stiffness approaches zero at the same finite value of $\delta$. This remarkable behavior needs to be explained by any theory that claims to describe the properties of chromium alloys. While we do not have an explanation for the correlations of the Figure at this time, we list here several ideas that may eventually lead to a theoretical description. First, the fact that, at higher rhenium concentrations, the ISDW-CSDW transition is first order [9] is consistent with a jump in $\delta$ over a forbidden range at that transition (but if there is such a jump, it has not 
been emphasized in publication), so current theoretical explanation of the firstorder nature of that transition [1] might possibly be able to explain the Figure, if extended to apply to it. Second, arguments have been published that crystallographic commensurate-to-incommensurate transitions must always be discontinuous, due to interactions between incommensurate domain walls.[10] Since little is known about ISDW domain walls, it is not clear if these arguments can be applied to them. Third, reduction of stiffness is softening, so the effect we are seeing may indicate a kind of "mode-softening" transition for $\delta \lesssim 0.017$ rlu. Normally, however, the mode involved is a real (underdamped) excitation with a dispersion relation that softens, whereas critical fluctuations are virtual (overdamped) excitations with a self-energy functional, $R(Q)$, which resembles but is not exactly a dispersion relation, that appears to be softening. Magnetic soft-mode transitions, as usually discussed, involve the magnetic interactions in a material generating a dispersion relation for a normally dispersionless (single-ion) "crystalline electric field" excitation above a nonmagnetic singlet ground state,[11] which then softens to cause magnetic ordering. No analogous excitation of nonmagnetic origin is known in chromium alloys.

This work was supported in part by US DOE grant DE-FG05-88ER45353 and the Natural Sciences and Engineering Research Council of Canada. Research at Brookhaven National Laboratory was supported by US DOE contract DE-AC02CH00016. 


\section{References}

[1] For reviews, see E. Fawcett, Rev. Mod. Phys. 60 (1988) 209, and E. Fawcett, H.L. Alberts, V.Y. Galkin, D.R. Noakes, and J.V. Yakhmi, Rev. Mod. Phys. 66 (1994) 25.

[2] H. Sato and K. Maki, Int. J. Magn. 6 (1974) 193.

[3] K. Machida and M. Fujita, Phys. Rev. B 30 (1984) 5284; R.S. Fishman and S.H. Liu, Phys. Rev. B 45 (1992) 12306 and 47 (1993) 11870.

[4] P. Hill, N. Ali, A.J.A. Oliviera, W.A. Ortiz, P.C. de Camargo and E. Fawcett, J. Phys. Cond. Matter 6 (1994) 1761.

[5] D.R. Noakes, E. Fawcett, T.M. Holden and P.C. de Camargo, Phys. Rev. Lett. 65 (1990) 369; E. Fawcett, T.M. Holden and D.R. Noakes, Physica B 174 (1991) 18; D.R. Noakes, E. Fawcett and T.M. Holden, Phys. Rev. B 55 (1997) 12504.

[6] K. Mikke and J. Jankowska, J. Phys. F 10 (1980) L159.

[7] E. Fawcett and D.R. Noakes, Int. J. Mod. Phys. B 7 (1993) 624.

[8] E. Fawcett and C. Vettier, J. Phys. (Paris) 43 (1982) 1365, see our Rev. Mod. Phys. 66 review (ibid), Fig. 38. 
[9] A.H. Boshoff, H.L. Alberts, P. de V. du Plessis and A.M. Vettier, J. Phys. Cond. Matter 5 (1993) 5353.

[10] J. Lajzerowicz, A.P. Levanyuk and S.A. Minyukov, Phys. Rev. B 54 (1996) 12073, and refs. cited therein.

[11] $\operatorname{Li}(\mathrm{Tb}, \mathrm{Y}) \mathrm{F}_{4}$ : R.W. Youngblood et al, Phys. Rev. Lett. 49 (1982) 1724; J. Kötzler et al., Phys. Rev. Lett. 60 (1988) 647; M. Grahl and J. Kötzler, J. Magn. and Magn. Mater. 104-107 (1992) 219; C. Marx et al., Phys. Lett. A 210 (1996) 141; uniaxially-strained praseodymium: K.A. McEwen et al., Physica B (1983) 152; $\mathrm{Pr}_{2} \mathrm{BaNiO}_{5}$ : A. Zheludev et al., Phys. Rev. B 54 (1996) 6437. 


\section{Figure Caption}

Correlations among Sato-Maki critical neutron scattering model parameters in chromium alloy single crystals just above $T_{N} . A^{2}$ is the magnetic stiffness, $\mathrm{r}^{2} / \chi^{0}$ is the reciprocal of the amplitude scale. The straight lines are guides for the eye. 


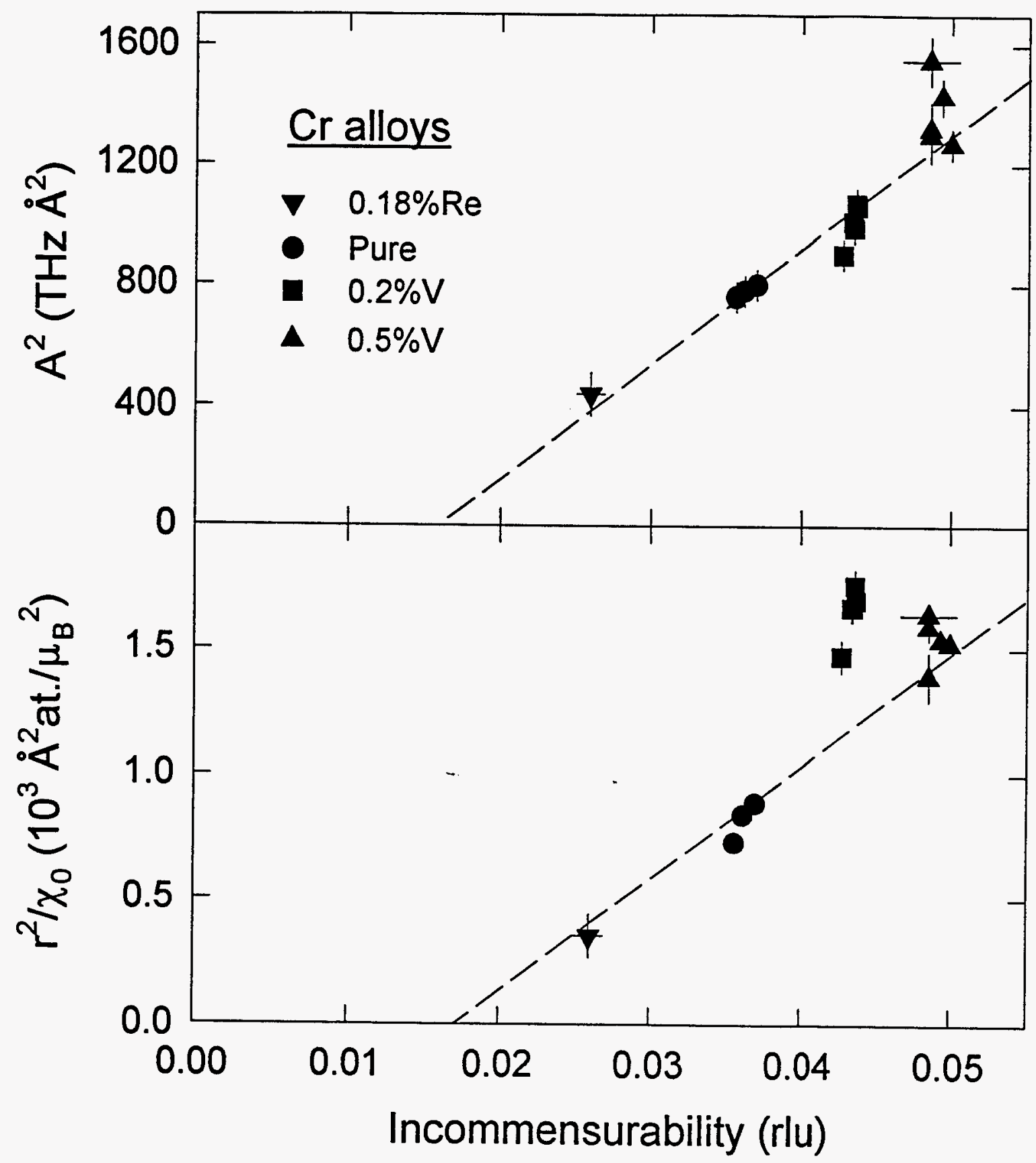

D.R. Nonkes of al so.? 
Report Number (14) BNL--64607 CONE-970814- -

Publ. Date (11)

Sponsor Code (18)

199707

UC Category (19)

DOEIER, XF

UC-4/0, DOE/ER 